\title{
Analyse tribo-énergétique du procédé de toilage : intervention des variables «process »-fréquence d'oscillation et temps de cycle sur les caractéristiques d'état de surface et de forme
}

\author{
Mohamed El Mansori ${ }^{1, a}$, Patrick Ghidossi ${ }^{1}$, Edoardo Sura ${ }^{2}$, Stéphane Deblaise $^{2}$ \\ et Tommaso Dal Negro ${ }^{2}$ \\ 1 Laboratoire de Mécanique et Procédés de Fabrication (LMPF-EA4106), ENSAM, Rue Saint Dominique, BP 508, \\ 51006 Châlons-en-Champagne, France \\ 2 Renault, Direction de la Mécanique/Direction de l'Ingénierie Process, 92508 Rueil-Malmaison Cedex, France
}

Reçu le 17 novembre 2005, accepté le 19 juin 2006

\begin{abstract}
Résumé - Le temps de cycle et la fréquence d'oscillation sont des variables « process » majeurs en technologies avancées de superfinition par toilage. Cet article examine leur rôle en liaison avec les mécanismes physiques activés (coupe, labourage ou glissement). On introduit ainsi une description énergétique du procédé de toilage. Celle-ci exploite la variable d'énergie dissipée pour étudier l'effet du temps de toilage et observer l'influence supposée d'alignement (oscillation axiale de la toile abrasive). Deux régimes de toilage sont identifiés et ainsi discutés.
\end{abstract}

Mots clés : Toilage / usure / abrasion / approche énergétique

Abstract - Tribo-energetic analysis of belt grinding process: variables process interfere - oscillation frequency and cycle-time grinding on surface characterization and form aspects. Cycletime and oscillation frequency are the most important variables in advanced technologies of superfinishing by belt grinding. This paper considers their role in connection with the activated physical mechanisms (cutting, ploughing or sliding). An energy description of the belt grinding process is introduced. The dissipated energy variable is considered to investigate the time-grinding dependant behaviour and to observe the possible influence of "tracking" (axial belt oscillation). Two "belt grinding regimes" are identified and then discussed.

Key words: Belt grinding / wear / abrasion / energy approach

\section{Introduction}

La compétition automobile internationale se place aujourd'hui dans un axe de réduction de consommation de carburant et d'augmentation de puissance spécifique des moteurs avec des émissions de $\mathrm{CO}_{2}$ limitées. Or, pour un moteur essence, les pertes par frottements (PMF) dues aux paliers vilebrequins s'élèvent entre 20 et $25 \%$ du total des pertes. Un gain sur ces frottements est un gain direct en émissions de $\mathrm{CO}_{2}$. Alors que l'ACEA (Association de Constructeurs Européens d'Automobiles) s'est engagée pour ramener les émissions de $\mathrm{CO}_{2}$ à $140 \mathrm{~g} . \mathrm{km}^{-1}$ en 2008 (165 g. $\mathrm{km}^{-1}$ atteint en 2004), les travaux sur la diminution des frottements sont un des axes privilégiés de l'industrie automobile [1]. Cette course à la performance moteur génère des exigences qualité des pièces usinées

a Auteur correspondant :

mohamed.elmansori@châlons.ensam.fr très élevées, ceci dans des contraintes de fabrication de forts volumes. Les spécifications des bureaux d'étude ont ainsi tendance à être de plus en plus sévères. Des intervalles de tolérance IT inférieurs à 10 microns sur des diamètres de $50 \mathrm{~mm}$, des états de surface avec des rugosités $R a<0,04$ microns, des défauts de forme inférieurs à 2 microns... Ces tolérances sévères sont obtenues par des procédés de finition et superfinition qui complètent ceux d'ébauche où les tolérances n'excèdent pas le centième de millimètres. L'association de tolérances sévères, de forts volumes de production et réduction des coûts, exige la parfaite maîtrise de la gamme de fabrication, en particulier pour la finition et la superfinition. Or, toute maîtrise des procédés de la phase de finition requiert deux composantes essentielles :

1. une forte connaissance du savoir-faire métiers par les ingénieurs de fabrication; 


\section{Nomenclature}

\begin{tabular}{|lll|}
\hline$A$ & Amplitude des oscillations axiales & $(\mathrm{mm})$ \\
$A r$ & Pas moyen de grain (pas moyen des motifs de rugosité) & $(\mu \mathrm{m})$ \\
$C$ & Circularité & $(\mu \mathrm{m})$ \\
$C_{0}$ & Circularité avant toilage & $(\mu \mathrm{m})$ \\
$C^{\mathrm{d}}$ & Circularité après toilage avec mâchoires rigides & $(\mu \mathrm{m})$ \\
$C^{\mathrm{s}}$ & Circularité après toilage avec mâchoires souples & $(\mu \mathrm{m})$ \\
$e_{\mathrm{p}}$ & Épaisseur de matière enlevée & $(\mathrm{mm})$ \\
$E_{\text {toilage }}$ & Énergie de toilage & $(\mathrm{J})$ \\
$E_{\mathrm{sp}}^{\mathrm{t}}$ & Énergie spécifique de toilage & $\left(\mathrm{J} . \mathrm{mm}^{-3}\right)$ \\
$f$ & Fréquence des oscillations axiales & $(\mathrm{Hz})$ \\
$F_{\mathrm{s}}$ & Effort de serrage & $(\mathrm{N})$ \\
$N_{\mathrm{r}}$ & Vitesse de rotation de la pièce & $\left(\mathrm{tr} . \mathrm{min}^{-1}\right)$ \\
$P_{0}$ & Puissance à vide & $(\mathrm{W})$ \\
$P_{\text {charge }}$ & Puissance en charge & $(\mathrm{W})$ \\
$\bar{P}_{0}$ & Puissance moyenne à vide & $(\mathrm{W})$ \\
$\bar{P}_{\text {charge }}$ & Puissance moyenne en charge & $(\mathrm{W})$ \\
$P_{\text {toilage }}$ & Puissance de toilage & $(\mathrm{W})$ \\
$Q_{\mathrm{m}}$ & Taux de matière enlevée & $\left(\mathrm{mm}{ }^{3} \cdot \mathrm{s}^{-1}\right)$ \\
$R$ & Rugosité superficielle & $(\mu \mathrm{m})$ \\
$t_{\mathrm{c}}$ & Temps caractéristique de coupe & $(\mathrm{s})$ \\
$t_{\mathrm{c}}^{\mathrm{d}}$ & Temps caractéristique de coupe avec mâchoires rigides & $(\mathrm{s})$ \\
$t_{\mathrm{c}}^{\mathrm{s}}$ & Temps caractéristique de coupe avec mâchoires souples & $(\mathrm{s})$ \\
$t_{\text {toilage }}$ & Temps de cycle & $(\mathrm{s})$ \\
$W$ & Ondulation superficielle & $(\mu \mathrm{m})$ \\
$W_{\text {toilage }}$ & Volume de matière enlevée & $\left.(\mathrm{mm})^{3}\right)$ \\
\hline
\end{tabular}

2. une compréhension approfondie des phénomènes physiques par les ingénieurs de conception des technologies de finition.

Dans son principe la technologie de superfinition par toilage est remarquablement simple et peu coûteuse. Malheureusement, sa réalisation pratique pose des problèmes difficiles en raison de la méconnaissance des processus physiques activés au cours du toilage. En effet dans l'industrie, la mise au point d'une gamme de toilage reste encore empirique issue d'un ensemble de règles pratiques qui s'apparentent plutôt à des « recettes ». Pour preuve, chaque nouvelle spécification d'état de surface sur un plan-produit se traduit, pour le process, par un travail important de codification de règles proposées sous forme de procédures et de tolérances, indispensable à une fabrication en série. Il est clair que, dans ce domaine, le lancement de campagnes d'essais à chaque nouvelle spécification avec des coûts de fabrication très élevés réduit les ressources projets. L'apport de la physique de l'abrasion permettrait sans doute de diminuer substantiellement les coûts de cette phase initiale et du temps de mise au point du «process ». Ceci est d'autant vrai que les variables « process » d'influence sont en effet extrêmement nombreuses, qu'elles s'interpénètrent de façon fort complexe et que, en somme les études réellement nouvelles intervenues scientifiquement depuis plusieurs décades sont quasi-inexistantes. Il suffit de regarder les dates auxquelles ont été publiées et proposées les définitions et règles qui régissent en partie les pratiques industrielles $[2,3]$. De plus, il n'a pas été trouvé dans la littérature d'études récentes et scientifiques traitant directement la technologie utilisée par l'alliance RENAULTNISSAN pour la superfinition des vilebrequins. Il existe sûrement quelques données et savoir-faire non publiés et propres à certaines entreprises de mécanique utilisant cette variété de procédés de finition avancés. En revanche, il existe de nombreuses publications sur l'abrasion à deux ou trois corps $[4,5]$ et quelques articles très récents sur le toilage $[6,7]$. Malheureusement leurs résultats n'ont pas pu être extrapolés au cas du toilage industriel dans le secteur automobile car les conditions de sollicitations sont trop différentes.

\section{Les variables « process $» d$ 'influence reconnues}

L'analyse ci-après s'appuie sur un boisseau de faits empiriques qui montre l'intervention critique de certaines variables « process » de toilage. Celles-ci prédominent, les unes ou les autres, selon l'opération d'usinage envisagée (ébauche ou finition). De cette prédominance, liée essentiellement aux amplitudes des mécanismes physiques activés à l'interface toile abrasive-pièce, dépendra en effet le niveau fonctionnel consécutif de la surface toilée. Énumérons ici quelques faits empiriques pour illustrer ce dessein :

1. Lorsqu'on presse la toile contre la surface avec un rouleau de contact (appelé mâchoire de toile ou mâchoire de travail) très rigide, ceci s'accompagne toujours [8-10] : 
- d'un usinage à taux d'enlèvement de matière élevé; - d'une qualité de finition ordinaire avec une faible tenue des sous-couches en fatigue.

Curieusement, lorsque une haute précision de la cote et une meilleure qualité de la forme géométrique de surface finie sont recherchées, des mâchoires avec des stries définies dans un angle par rapport à la direction d'abrasion compris entre $90^{\circ}$ et $45^{\circ}$, sont fréquemment utilisés $[2,8]$. Selon l'application et la qualité de finition exigée, la dureté des mâchoires de travail est fondamentale. Pour l'ébauche (procédé de pré-finition), on utilise généralement des mâchoires rigides permettant un enlèvement de matière relativement important. Pour des raisons de résistance à l'usure et de longévité, les mâchoires de travail revêtues en couches de céramique spéciale sont utilisées. Lors de la finition (phase ultime), les mâchoires de travail souples s'imposent lorsqu'il s'agit d'améliorer significativement les valeurs superficielles et d'augmenter le taux de portance $[2,9]$. Ainsi, lorsqu'on met en parallèle le résultat apparent d'une simple modification de la dureté des mâchoires de toile et son incidence sur la qualité de finition ; on s'aperçoit que malgré son intérêt fondamental, l'action de cette grandeur (dureté de mâchoire) reste encore confuse et imprécise. Ceci probablement parce qu'on ignore les incidences sur les conditions locales du contact film abrasif-pièce, les rôles respectifs de l'élasticité et de la plasticité lors de l'abrasion $[7,11]$.

2. Lorsqu'on conjugue la rotation du cylindre de contact et l'oscillation axiale du film abrasif lors du toilage, le parachèvement des géométries de pièces de haute précision avec de tolérances serrées est obtenu $[2,7,8]$. Ceci aujourd'hui est commun à tous les procédés de superfinition par toilage où l'outil oscillant à une fréquence comprise entre 2 et $85 \mathrm{~Hz}$, est comprimé par une pression constante de 9 à 40 N.cm ${ }^{-2}$ environ contre la surface de la pièce en rotation. Le principe d'oscillation est même parfois utilisé en rectification avec traits croisés permettant d'obtenir des géométries de pièce planes et sphériques très précises [8]. L'hypothèse avancée dans ce cas précis afin d'expliquer cette précision est l'augmentation du nombre de grains actifs obtenu en faisant vibrer la meule sur la pièce $[7,9]$. La composante de coupe domine ainsi celle du labourage en conséquence de quoi l'état de surface se trouve amélioré. Précisons ici que cette interprétation n'a pas encore été prouvée de façon certaine en meulage et la question reste pleinement posée dans le cas de la superfinition par toile.

3. Lorsqu'un indexage discontinu de la toile est appliqué, la reproductibilité et la constance des résultats du toilage sont maximales $[2,8,9]$. Cela signifie que l'on maîtrise au préalable le temps de cycle de sorte que l'avance de la toile puisse être adaptée de manière optimale à chaque diamètre d'usinage. Ceci permet aussi de réduire la consommation inutile de la toile et d'obtenir les conditions de départ pour chaque nouvelle pièce, condition indispensable à l'obtention de résultats reproductibles. L'ennui est que l'influence de la variable « process » temps de cycle est encore insuffisamment connue. Quels sont en effet les facteurs qui accroissent ou réduisent le temps de cycle et par voie de conséquence, quels sont les facteurs d'usinage à la toile qui sont les plus favorables?

Bien entendu, on pourrait encore multiplier les faits expérimentaux qui témoignent du rôle décisif des effets corrélatifs encore méconnus des variables «process » et de la fonctionnalité des surfaces finies par toilage. Notons par ailleurs, qu'il est difficile de décrire à ce jour l'enchaînement cause/conséquence des phénomènes de toilage car, hormis l'influence des paramètres «process », d'autres effets structurels se superposent. Ces corrélations qui ne sont donc ni généralisables ni prédictives viennent démontrer la nécessité de ce travail. L'objectif ici est de faire apparaître les mécanismes qui régissent l'intervention des variables « process », temps de cycle et fréquence d'oscillation, lors du toilage ébauche et de finition. À cette fin, des tests énergétiques de toilage industriel pour la superfinition de vilebrequins ont été mis au point.

\section{Démarche expérimentale}

La démarche développée ici pour contourner les difficultés d'étude de l'interface oscillante film abrasif/pièce consiste en une approche énergétique qui s'appuie sur :

1. des essais de toilage instrumentés où les valeurs effectives des efforts de serrage et des énergies mises en jeu (via des mesures de puissance) font partie des informations indispensables, dans un premier temps, à la description approchée du tribosystème toile/pièce.

2. des expertises de retour d'essais exploitées comme un traceur des conditions du contact film abrasif/pièce pour comprendre son fonctionnement afin de pouvoir, dans un second temps, en modéliser le comportement.

\subsection{Couple outil toile-pièce}

L'outil de toilage consiste en une toile abrasive de superfinition $3 \mathrm{M}$ référencée $372 \mathrm{~L}$. La figure 1 donne un aperçu microscopique de la disposition des grains d'abrasif appliqués sur film polyester. Elle précise aussi les caractéristiques dimensionnelles et superficielles de la toile utilisée. Les grains d'abrasif sont en oxyde d'aluminium avec une grosseur moyenne d'environ $30 \mu \mathrm{m}$. La distance inter-grains, définie par le paramètre $A r$, est relativement importante $(\sim 195 \mu \mathrm{m})$ afin d'assurer le logement des micro-copeaux générés en début de toilage.

Les surfaces toilées correspondent à des portées de vilebrequins (cf. Fig. 2). Leur dureté superficielle après traitement thermique est de $50 \mathrm{HRC}$ environ. La figure 2 précise aussi les paramètres de départ des éprouvettesvilebrequins avant toilage. 


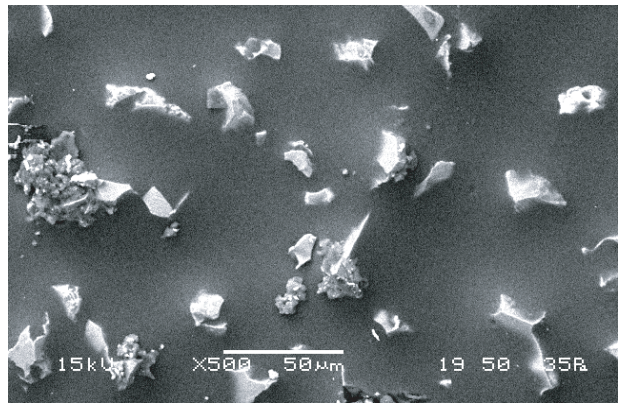

(a)

\begin{tabular}{|ll|}
\hline Producteur : & $3 \mathrm{M}$ \\
Type : & Microfinishing $372 \mathrm{~L}$ \\
*Matière des grains : & $\mathrm{AlO}$ \\
*Taille des grains : & $30 \mu \mathrm{m}$ \\
*Largeur : & $19,84 \mathrm{~mm}$ \\
Rugosité moyenne : & $\boldsymbol{R}=18 \mu \mathrm{m}$ \\
Distance inter-grains : & $\boldsymbol{A r}=195 \mu \mathrm{m}$ \\
\hline
\end{tabular}

* Fournies par $3 M$

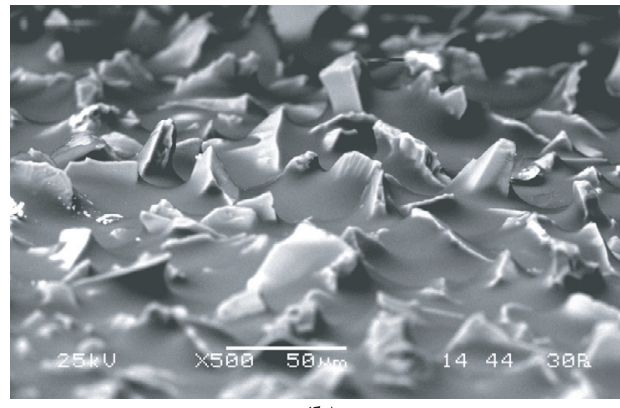

(b)

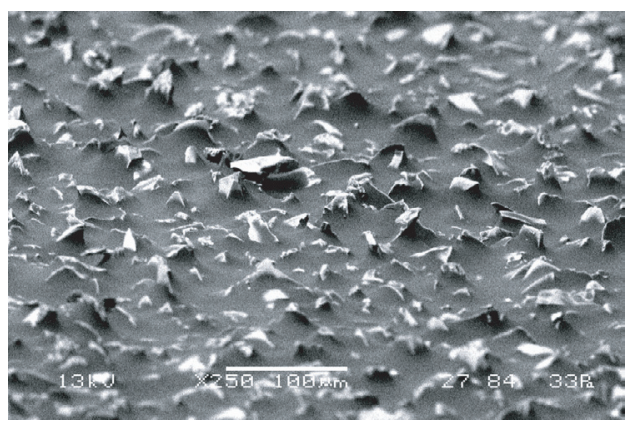

(c)

Fig. 1. Structure de la toile abrasive neuve avec les caractéristiques de départ (grossissement et inclinaison des observations : a) $\times 500,0^{\circ}$; b) $\times 500,5^{\circ}$; c) $\times 250,5^{\circ}$ ).

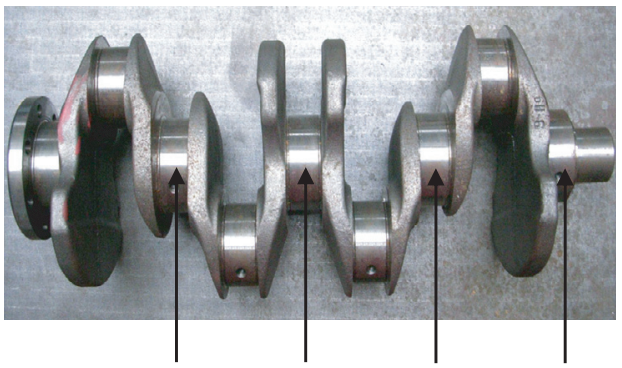

Les quatre portées de vilebrequin à usiner

\begin{tabular}{|ll|}
\hline Fournisseur : & Renault \\
Matériau : & Acier D38 MSV5S \\
Traitement thermique : & Induction \\
Opération pré-toilage : & Rectification \\
Diamètre des portées : & $54,795^{ \pm 0,01} \mathrm{~mm}$ \\
Largeur des portées : & $19,6^{ \pm 0,5} \mathrm{~mm}$ \\
Rugosité : & $0.5<\boldsymbol{R}<1.6 \mu \mathrm{m}$ \\
Ondulation : & $0.5<\boldsymbol{W}<0.8 \mu \mathrm{m}$ \\
Circularité : & $\boldsymbol{C}<5 \mu \mathrm{m}$ \\
Dureté superficielle : & $50 \mathrm{HRC}$ \\
\hline
\end{tabular}

Fig. 2. Exemple d'éprouvette-vilebrequin avec les paramètres de départ.

\subsection{Conduite des tests énergétiques de toilage}

Le banc d'essai (cf. Fig. 3) consiste en un tour conventionnel (puissance de $9 \mathrm{~kW}$ à la broche) équipé d'un appareil de superfinition de type Bader avec un bras d'usinage. Ceci conduit à une architecture horizontale de superfinition par toile, architecture habituellement utilisée pour la superfinition de portées cylindriques (vilebrequins). Le banc d'essai reproduit le toilage industriel selon lequel la toile abrasive est pressée par vérin pneumatique sur la périphérie du palier usiné par l'intermédiaire de deux mâchoires pendant un temps de cycle $t_{\text {toilage }}$ prédéfini. Chaque mâchoire est aussi constituée de cinq inserts (cf. Tab. 1) assurant l'enroulement total et le contact de la bande abrasive sur la surface superfinie, en couvrant un angle de $160^{\circ}$. L'effort de serrage des mâchoires $\left(F_{\mathrm{s}}\right)$ est de $400 \mathrm{~N}$. Il est mesuré par un capteur piézo-électrique Kistler type 9011A. La superfinition est assurée par deux mouvements combinés de rotation de la portée usinée ( $N_{\mathrm{r}}$ tour par minute) et d'oscillation des deux mâchoires comprimant la bande abrasive (dans le sens axial de la pièce à la fréquence $f$ et avec une amplitude d'oscillation $A$ ).

Deux campagnes d'essais ont été réalisées :

- la première explicite le mode d'intervention de la variable « process » durée de toilage ou temps de cycle : $t_{\text {toilage }}$

- la seconde montre l'influence de la fréquence d'oscillation du contact toile-pièce : $f$.

Tous les essais de toilage ont été réalisés à sec, sans avance de toile. La puissance absorbée par le processus de toilage est évaluée par la différence entre la puissance moyenne en charge $P_{\text {charge }}$ et celle à vide $P_{0}$ de la machine-outil (tour). Son acquisition in situ s'effectue par le biais d'un transducteur de puissance Digital 


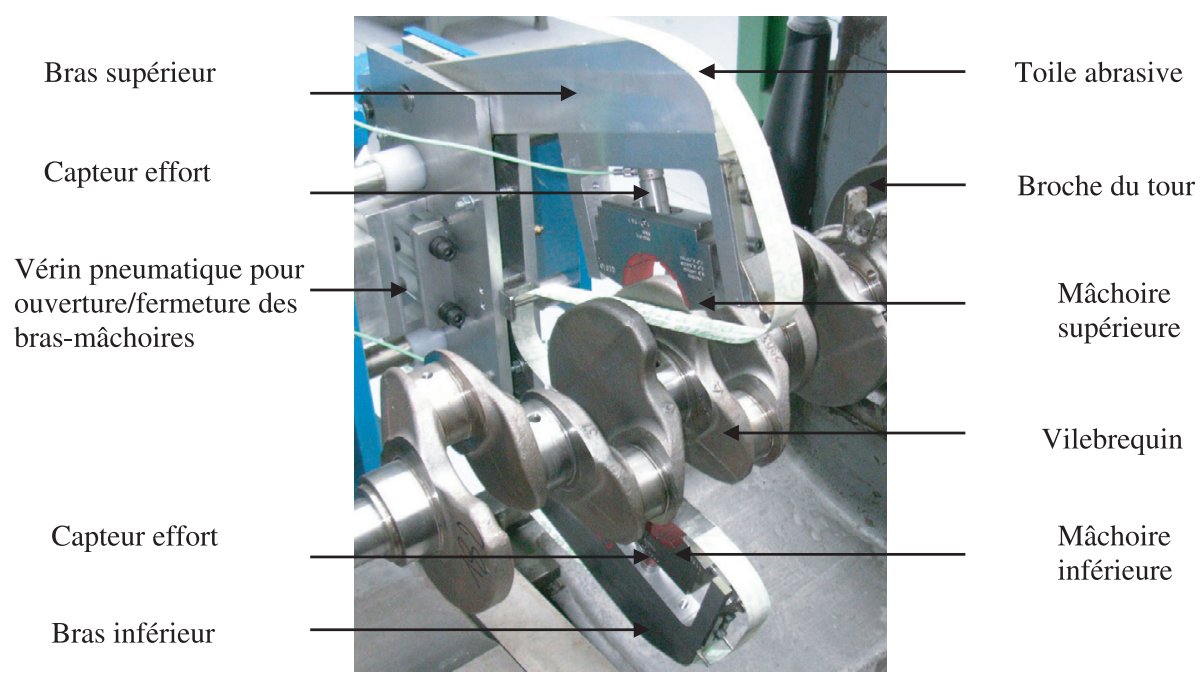

Fig. 3. Le banc de toilage utilisé pour la superfinition des portées de vilebrequins.

Tableau 1. Caractéristiques des inserts des mâchoires de toile.

\begin{tabular}{lcc}
\hline & Mâchoires rigides & Mâchoires souples \\
\hline Matériau d'inserts : & Grains corindon & Courbhane \\
Dureté : & $9 \mathrm{Mohs}$ & 85 Shore \\
Nombre d'inserts : & 5 & 5 \\
Distance entre deux inserts : & $1,8 \mathrm{~mm}$ & $1,8 \mathrm{~mm}$ \\
Rayon intérieur de courbure : & $54,79^{ \pm 0,1} \mathrm{~mm}$ & $54,79^{ \pm 0,1} \mathrm{~mm}$ \\
Longueur tangentielle : & $14 \mathrm{~mm}$ & $14 \mathrm{~mm}$ \\
Épaisseur radiale : & $0,5 \mathrm{~mm}$ & $0,5 \mathrm{~mm}$ \\
Largeur axiale : & $20 \mathrm{~mm}$ & $20 \mathrm{~mm}$ \\
\hline
\end{tabular}

Way-16 KW associé au logiciel de traitement Tool Power Analyser. Les variations du diamètre et de la circularité des portées toilées sont mesurées en utilisant une machine 2D (Adcole Model 1100). L'analyse de la rugosité obtenue en fonction des paramètres « process » s'appuie sur des mesures d'états de surface (sur profilomètre 3D Surfascan) avant et après toilage. Ces analyses sont complétées par des observations au microscope électronique à balayage (M.E.B.). Chaque essai de toilage est répété cinq fois et les résultats reportés dans ce travail sont validés par un test statistique (test Shapiro-Wilk).

\section{Performances comparées des variables «process »}

\subsection{Temps de cycle}

La figure 4 montre l'évolution de l'épaisseur de matière enlevée $e_{\mathrm{p}}$ en fonction du temps de cycle et ce pour les deux mâchoires de toile. Elle fait ressortir deux faits marquants :

- bien que des dispersions plus ou moins importantes soient observées, l'enlèvement de matière s'effectue essentiellement lors des premières secondes de toilage correspondant à un temps caractéristique de coupe $t_{\mathrm{c}}$. Celui-ci est respectivement de l'ordre de $t_{\mathrm{c}}^{\mathrm{s}}=6 \mathrm{~s}$ et

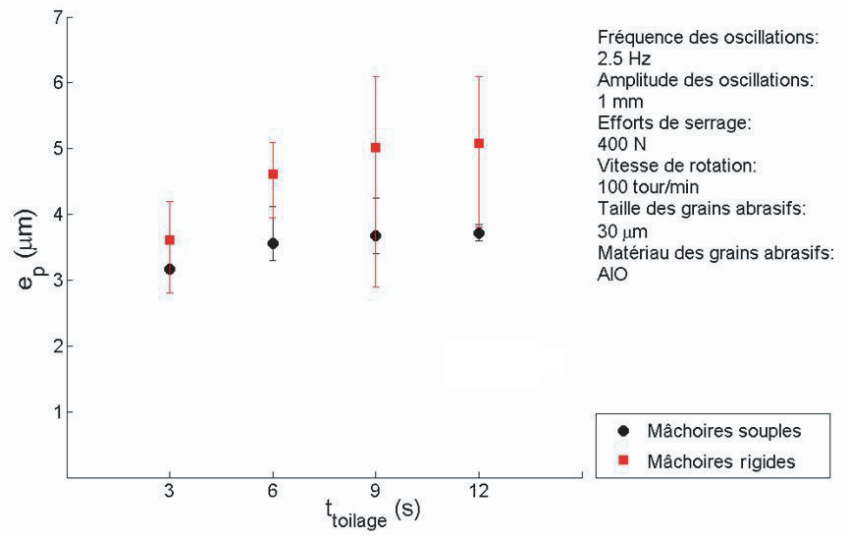

Fig. 4. Épaisseur de matière enlevée $\left(e_{\mathrm{p}}\right)$ en fonction du temps de cycle $\left(t_{\text {toilage }}\right)$ pour les deux mâchoires testées.

$t_{\mathrm{c}}^{\mathrm{d}}=9 \mathrm{~s}$ pour les mâchoires souples et rigides. Pour un temps de cycle $t_{\text {toilage }}>t_{\mathrm{c}}$, l'épaisseur de matière se situe dans une zone stable à variation quasi-nulle;

- lorsqu'il y a augmentation de la dureté des mâchoires de toile (mâchoires souples $\rightarrow$ mâchoires rigides), il y a génération d'un micro-enlèvement de matière significatif $(3,5 \mu \mathrm{m} \rightarrow 5 \mu \mathrm{m})$.

Par ailleurs, il a pu être vérifié que le prolongement du temps de cycle ne modifie pas la valeur minimale 


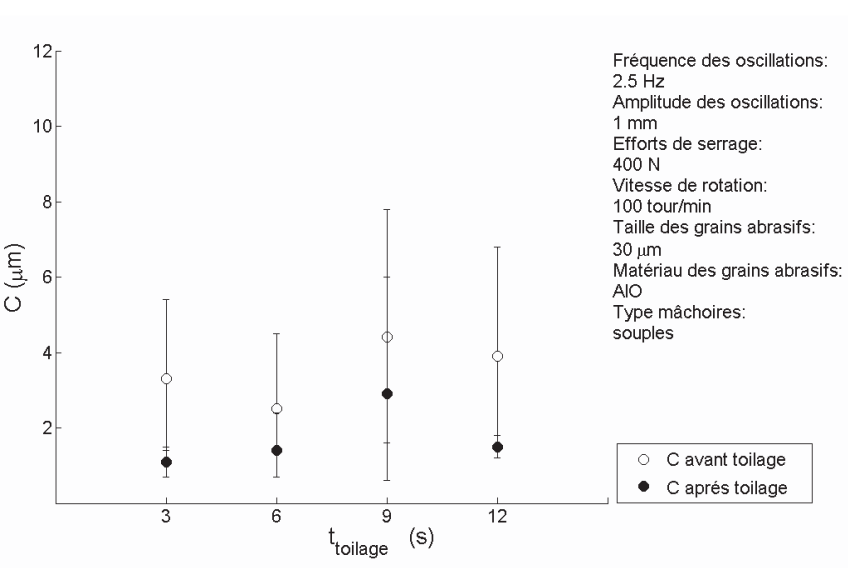

(a)

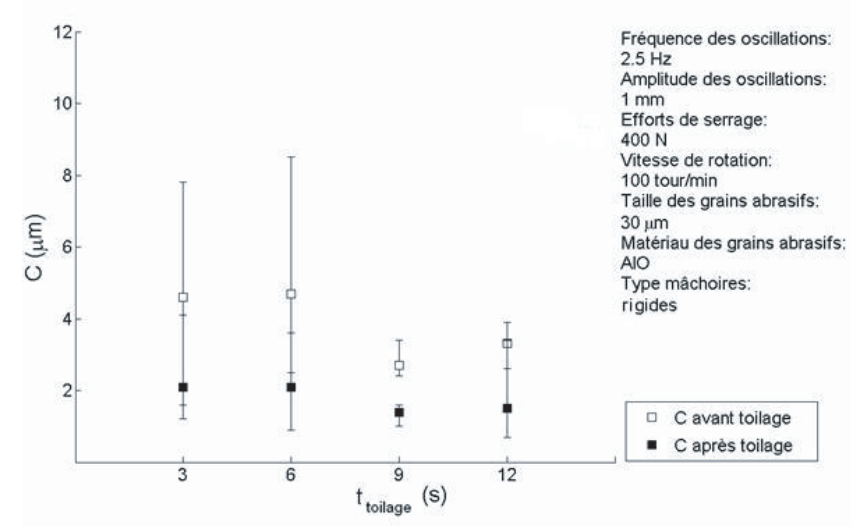

(b)

Fig. 5. Les variations de la circularité $(C)$ avec la durée de toilage $\left(t_{\text {toilage }}\right)$ pour les mâchoires souples (a) et rigides b).

d'écart de forme exprimé en terme de circularité, comme le montre la figure 5 . Celle-ci est obtenue après un temps cycle de 3 s et n'évolue pas avec le temps de toilage. Ceci est vrai aussi bien pour les mâchoires rigides que souples. Toutefois, on ne doit rien conclure sur le gain en circularité compris entre toilage avec mâchoires rigides et souples car la circularité est un indicateur trop « macroscopique ». Le gain en circularité comparé entre mâchoires rigides et souples n'est pas un indicateur fiable à cause des aspects très variés que constitue une circularité (cf. Fig. 6). En effet, les mâchoires rigides sont plus favorables à la réduction de circularité que celles souples car la rectification a pour fonction de :

- augmenter la précision de la cote « diamètre » (nonaméliorée par le toilage);

- réduire le défaut de forme macroscopique, c'est-à-dire l'aspect ovale (non-corrigé par le toilage).

Les performances en terme d'ondulation, de rugosité et de profondeur affectée en fonction du temps de cycle sont reportées sur la figure 7 . On peut en tirer les principales remarques suivantes:

- la dimension des irrégularités de surface, quantifiée ici par le critère de rugosité $R$ (NF ENCSO 12085),

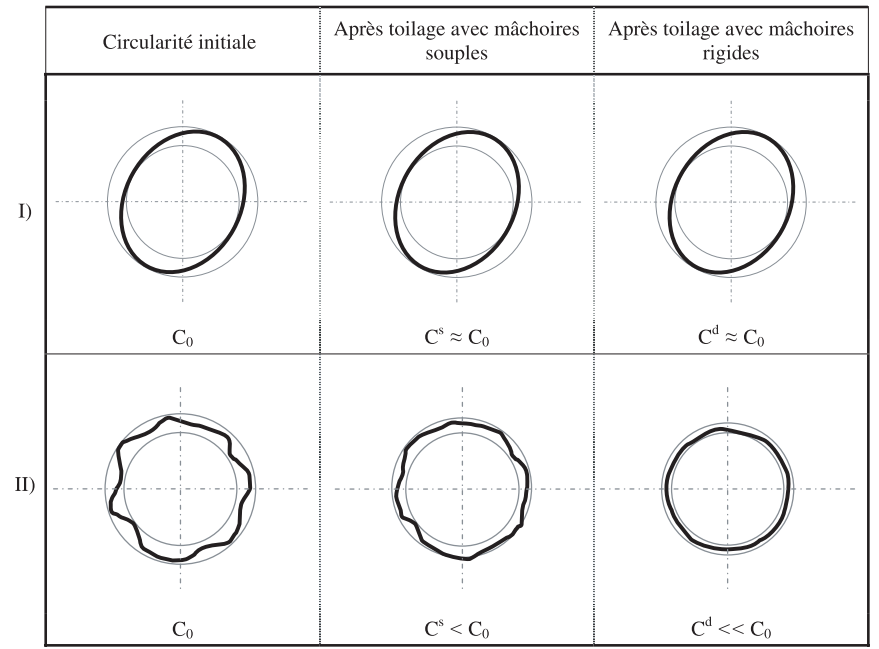

Fig. 6. Influence du toilage sur la circularité : le type de défaut I ne peut pas être corrigé par toilage. À l'inverse celui du type II peut être amélioré par toilage car il est sensible à la dureté des mâchoires de toile.

diminue considérablement après toilage. Ce procédé permet en effet d'obtenir très rapidement des pièces avec une faible rugosité.

- La variation des irrégularités de surface avec le temps de cycle montre la présence de deux régimes distincts :

1. régime primaire transitoire caractérisé par une nette diminution de $R$ (environ $60 \%$ ) pour des durées de toilage $t_{\text {toilage }}<6 \mathrm{~s}$;

2. régime secondaire établi où $R$ n'évolue plus. Remarquons aussi que les dispersions des valeurs de rugosité après toilage sont quasi-nulles.

- Les deux mâchoires de toile testées ont des performances comparables en terme de rugosité obtenue (0,3 $\mu \mathrm{m}$ obtenue avec les mâchoires rigides contre $0,25 \mu \mathrm{m}$ avec celles souples).

\subsection{Fréquence d'oscillation}

La fréquence d'oscillation influe non seulement sur le taux de matière enlevée mais également sur la diminution des irrégularités de surface de la pièce finie. Examinons graphiquement ces deux observations. Si l'on porte en abscisse la fréquence d'oscillation et en ordonnée l'épaisseur enlevée, et ce pour les deux mâchoires testées, on obtient les courbes de la figure 8. On note ici que sans oscillation de la toile abrasive $(f=0 \mathrm{~Hz})$, l'épaisseur reste à un niveau faible par rapport à celle obtenue aux fréquences supérieures. Ce bénéfice ira en s'amenuisant et on retrouve un niveau de matière enlevée similaire dès que $f>0$. Remarquons aussi que le taux de matière enlevé dépend de la dureté des mâchoires utilisées. En terme de rugosité, la figure 9 donne l'évolution du critère $R$, avant et après toilage, en fonction de la fréquence d'oscillation. Deux faits sont observés :

- d'une part la diminution du paramètre $R$ dès que $f>$ 0 et ceci reste vrai pour les deux mâchoires de travail ; 


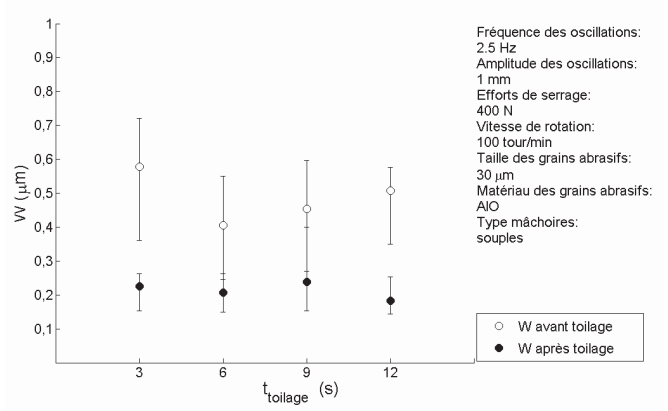

(a)

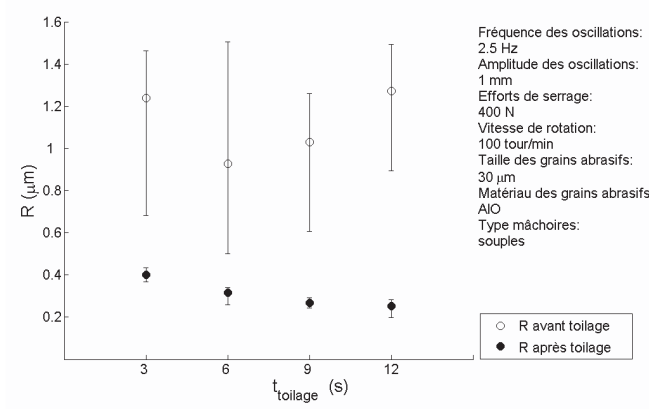

(c)

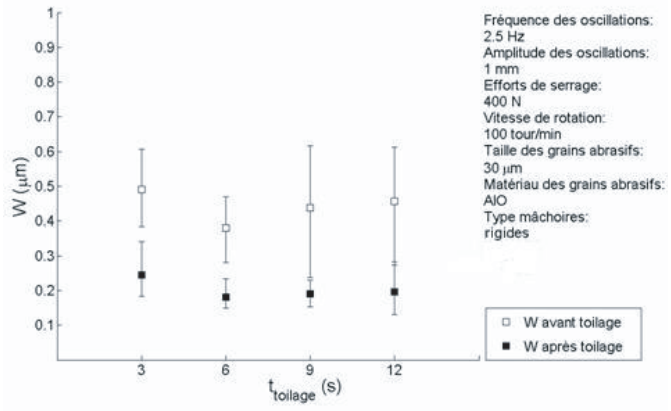

(b)

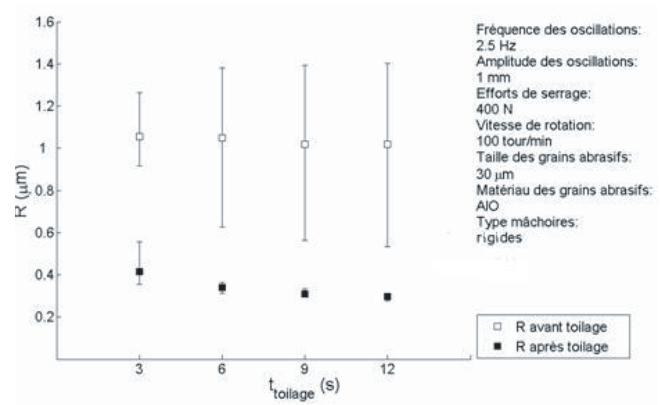

(d)

Fig. 7. Les variations de l'ondulation $(W)$ et de la rugosité superficielle $(R)$ avec le temps de cycle $\left(t_{\text {toilage}}\right)$ pour les mâchoires souples a) et c) et rigides b) et d).

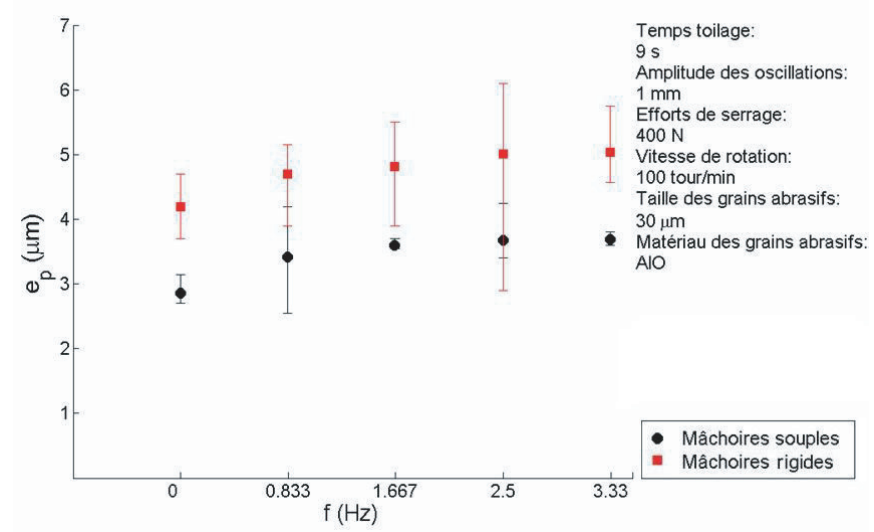

Fig. 8. Épaisseur de matière enlevée $\left(e_{\mathrm{p}}\right)$ en fonction de la fréquence d'oscillation axiale $(f)$ pour les deux mâchoires de travail.

- d'autre part, la dispersion diminue d'une manière très significative et surtout à des fréquences élevées.

Si les surfaces finies de la pièce après toilage sont améliorées à fréquence d'oscillation donnée, on constate que les caractéristiques de forme (ondulation et circularité) tendent à rejoindre celles obtenues sans oscillation (cf. Fig. 10). Il est clair que, en dehors du fait bénéfique relatif à l'atténuation des dispersions, l'effet de la variable « fréquence d'oscillation » est ici insignifiant.

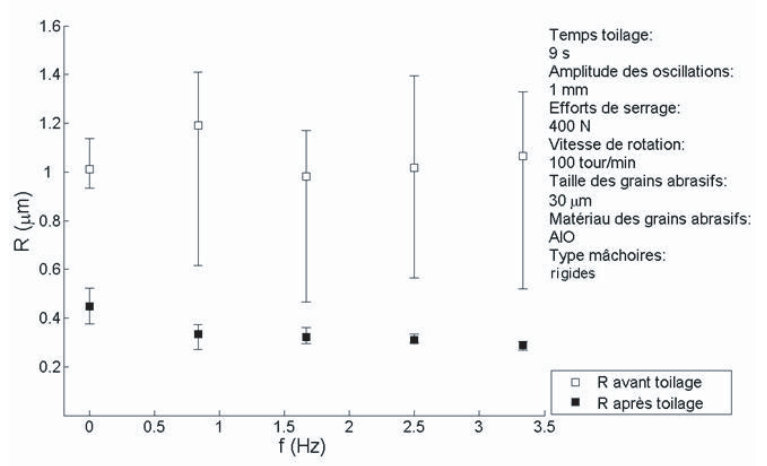

(a)

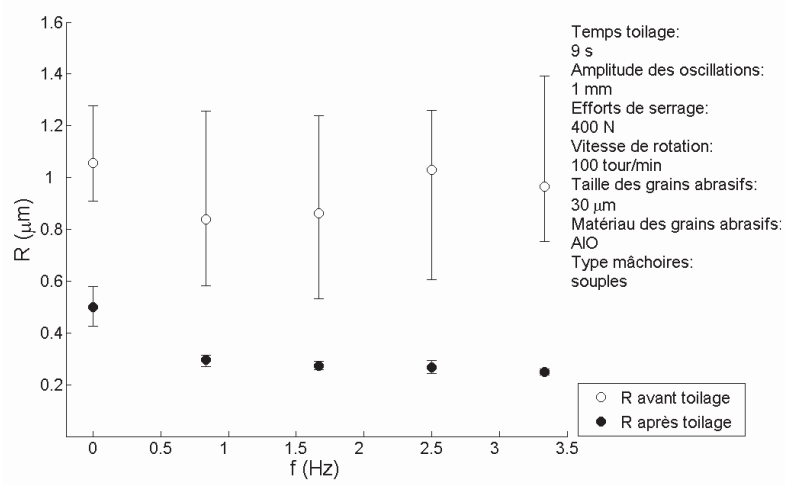

(b)

Fig. 9. Les variations de la rugosité $(R)$ avec la fréquence d'oscillation $(f)$ pour les mâchoires rigides a) et souples b). 


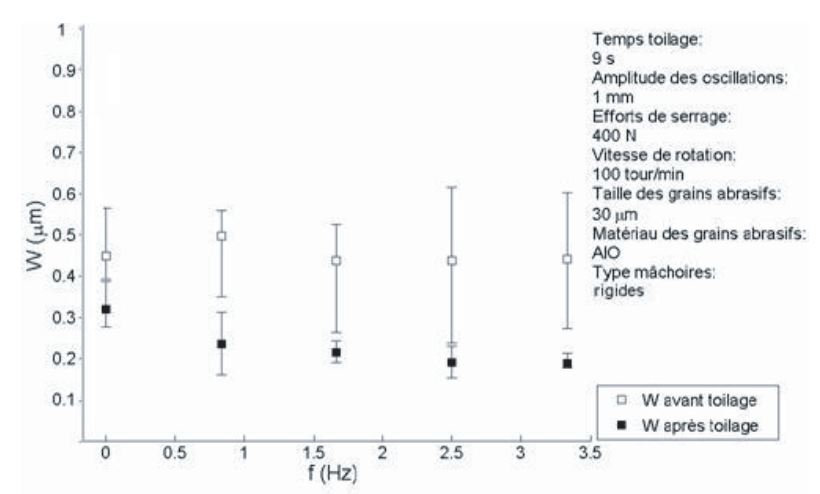

(a)

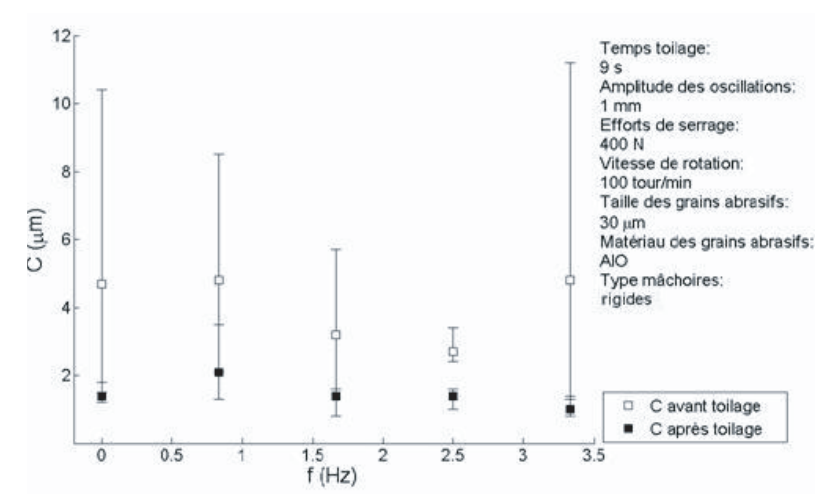

(c)

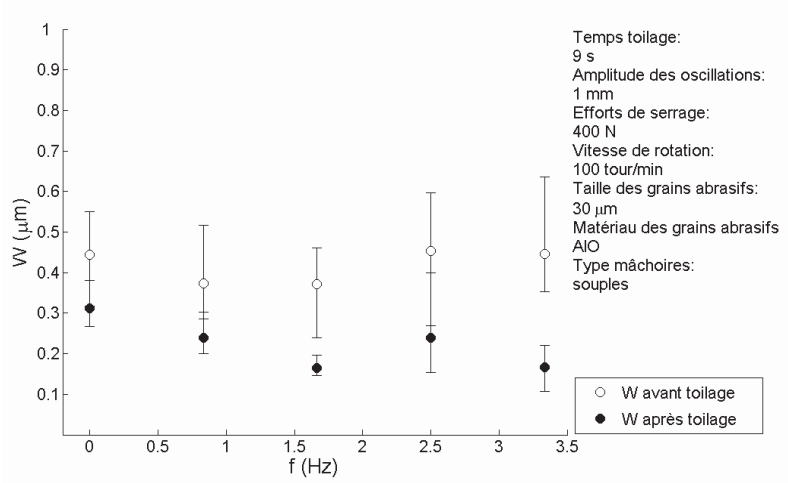

(b)

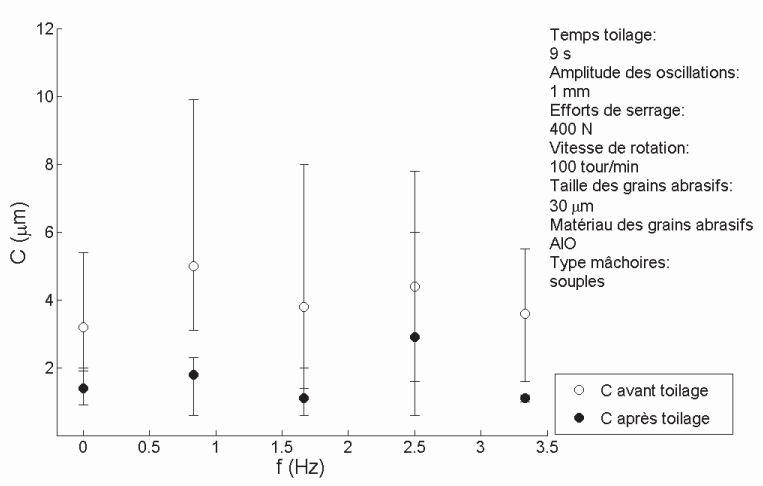

(d)

Fig. 10. Les variations de l'ondulation $(W)$ et de la circularité $(C)$ avec la fréquence des oscillations axiales $(f)$ pour les deux mâchoires de toile, rigides a) et c) et souples b) et d).

\section{5 État de l'abrasif comme traceur des conditions locales de toilage}

La caractéristique principale de la toile est constituée par sa morphologie : ceci implique à la fois l'angularité des grains (leur pouvoir de pénétration et de coupe) et la distribution de leur orientation géométrique au cours du processus de toilage (densité active des grains d'abrasif). Ainsi, le mode d'endommagement de l'abrasif, en liaison avec les paramètres «process », constitue un indicateur non-négligeable dans la précision de leur influence.

\subsection{Influence du temps cycle}

La figure 12 montre l'évolution de l'état de la toile abrasive en fonction du temps de cycle. Les formes micrographiques, ont été réalisées au MEB, par visée avec une inclinaison à $0^{\circ}$ par rapport à la surface de la toile abrasive. Comme le montre la figure 1 , la bande neuve porte des grains d'abrasif orientés, de taille moyenne $30 \mu \mathrm{m}$, qui présentent à la surface leur meilleure aptitude à la coupe (angularité $\approx 15 \mu \mathrm{m}$ ). De plus, l'espacement entre les grains est suffisamment grand pour permettre le logement de micro-copeaux (cf. Fig. 1). La vue 3D de la

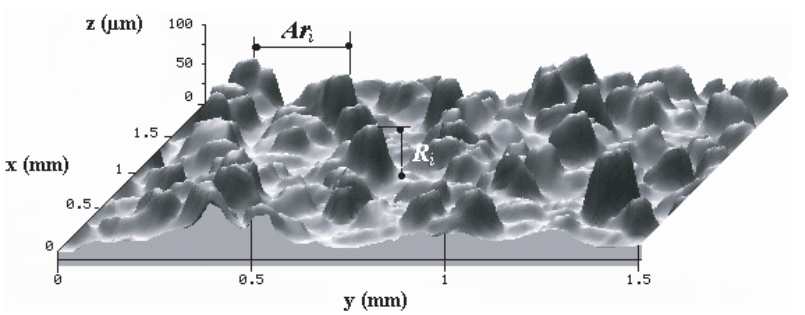

Fig. 11. Vue 3D du film abrasif $3 \mathrm{M}$ neuf avec une granulométrie moyenne de $30 \mu \mathrm{m}$.

figure 11 montre cet effet. Le paramètre $A r$ ici est une image de la valeur du logement des copeaux, le paramètre $R$ étant l'image du pouvoir de pénétration d'un grain dans la surface toilée. Après seulement trois secondes de toilage (Fig. 12), la toile présente une surface entièrement recouverte de micro-débris. La couche de transfert est constituée de micro-copeaux libres ou logés dans la structure de la toile. Le prolongement du temps cycle conduit à la fragmentation des micro-copeaux puis à la formation de conglomérats de particules broyées et fortement compactées. Le processus de fragmentation, agglomération de particules broyées et évacuation de la couche de transfert, conduit à l'instabilité dans le mode d'usinage par 

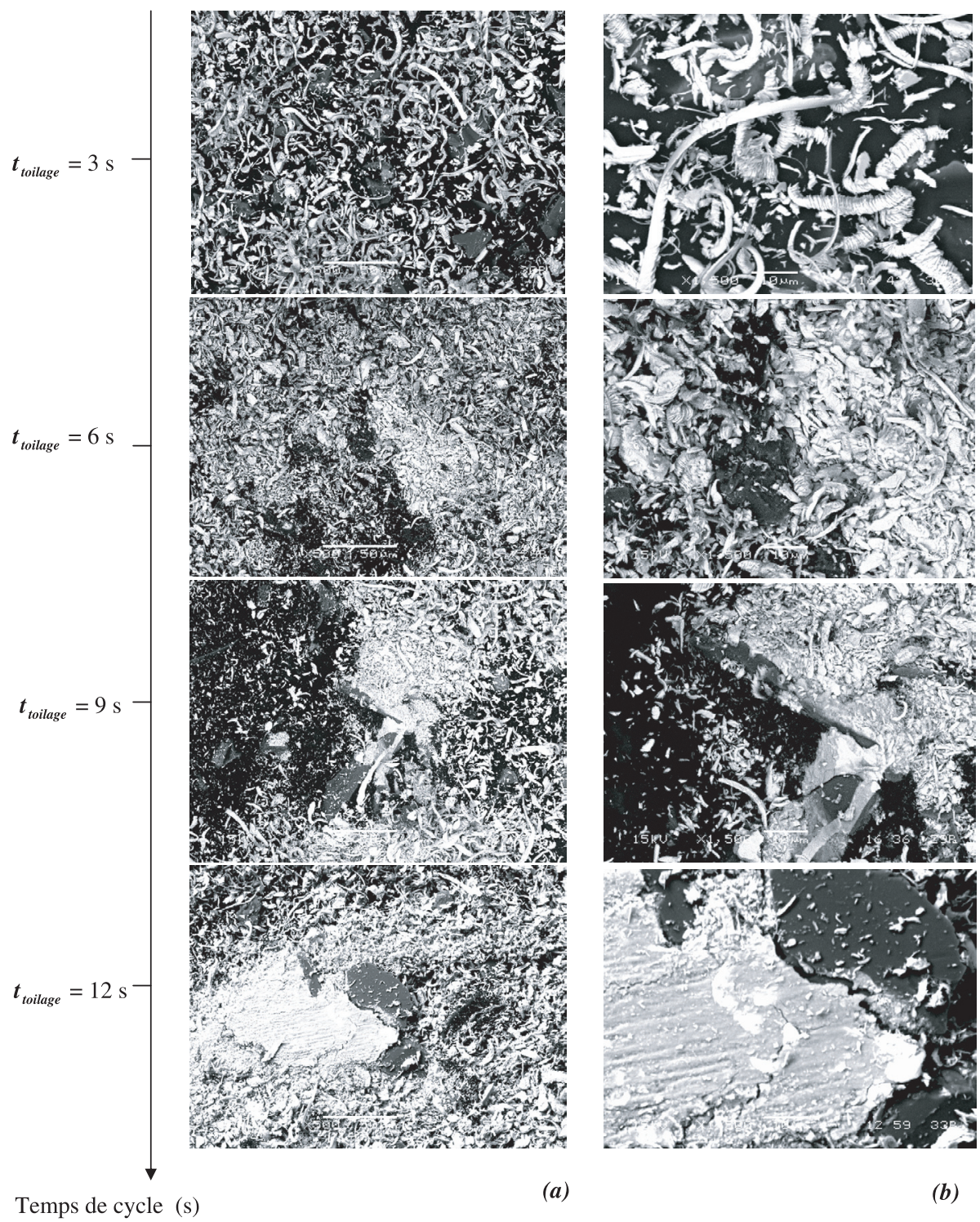

(a)

(b)

Fig. 12. Évolution des micro-débris logés dans la toile abrasive en fonction de la durée de toilage $t_{\text {toilage }}$ (grossissement $: \times 500$ a) et $\times 1500 \mathrm{~b})$, inclinaison des observations : $\left.0^{\circ}\right) .\left(f=2,5 \mathrm{~Hz}, A=1 \mathrm{~mm}, F_{\mathrm{s}}=400 \mathrm{~N}, N_{\mathrm{r}}=100\right.$ tr.min $^{-1}$, taille des grains abrasifs : $30 \mu \mathrm{m}$, matériau des grains abrasifs : AlO, type de mâchoires : rigides.)

toilage. L'action de la toile abrasive passe de la rainure par émission de micro-copeau au sillon de déformation plastique (labourage). Il y a perte de fonctionnalité de la toile qui n'est pas due à une usure des grains d'abrasif mais plutôt à la saturation de logement de micro-copeaux. On voit bien ici que l'avance de toile et l'arrosage de fluide de coupe auront un rôle pertinent dans la qualité de finition par film abrasif. Afin de confirmer la perte de fonctionnalité de la toile par effet de saturation (pouvoir de logement), des observations complémentaires de la toile après nettoyage ont été effectuées. La procédure de nettoyage consiste à appliquer un adhésif sur la bande abrasive en fin de toilage afin de décoller la couche de transfert et de logement des micro-débris. La toile est mise ensuite en suspension dans de l'alcool puis séchée par ondes ultrasons. Cette technique permet par ailleurs d'accéder aux micro-copeaux primaires, c'est-à-dire, ceux formés en début de toilage. La figure 13 résume les observations de l'évolution de la structure des grains d'abrasif de la toile après nettoyage en fonction de la durée de toilage $t_{\text {toilage }}$. Même si le pouvoir de coupe de la toile diminue (l'angularité chute à environ $8 \mu \mathrm{m}$ ) avec un émoussage visible des arêtes vives, elle conserve cependant une structure fonctionnelle.

\subsection{Influence de la fréquence des oscillations}

Le paramètre «process » fréquence d'oscillation influence simultanément le mode de fragmentation des micro-débris de transfert ainsi que la densité des microcopeaux logés dans la toile abrasive (Fig. 14). Les 


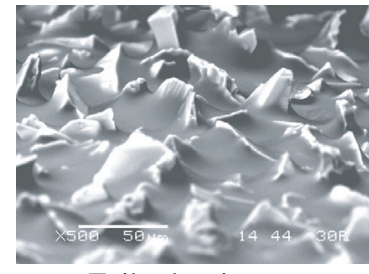

Toile abrasive neuve

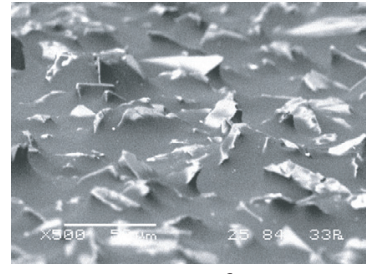

$t_{\text {toilage }}=3 \mathrm{~s}$

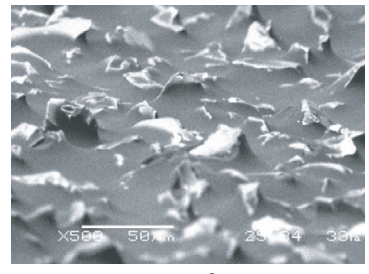

$t_{\text {toilage }}=6 \mathrm{~s}$
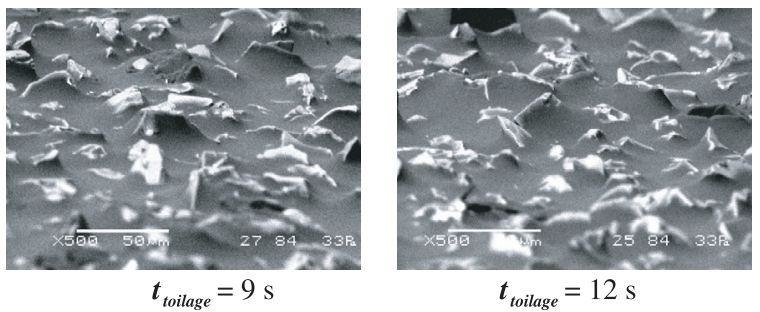

Fig. 13. Morphologie évolutive des grains d'abrasif de la toile après nettoyage en fonction de la durée de toilage $t_{\text {toilage }}$ $\left(f=2,5 \mathrm{~Hz}, N_{\mathrm{r}}=100 \mathrm{tr} \cdot \mathrm{min}^{-1}, F_{\mathrm{s}}=400 \mathrm{~N}, A=1 \mathrm{~mm}\right.$, taille des grains abrasifs : $30 \mu \mathrm{m}$, matériau des grains abrasifs : AlO, type mâchoires : rigides).

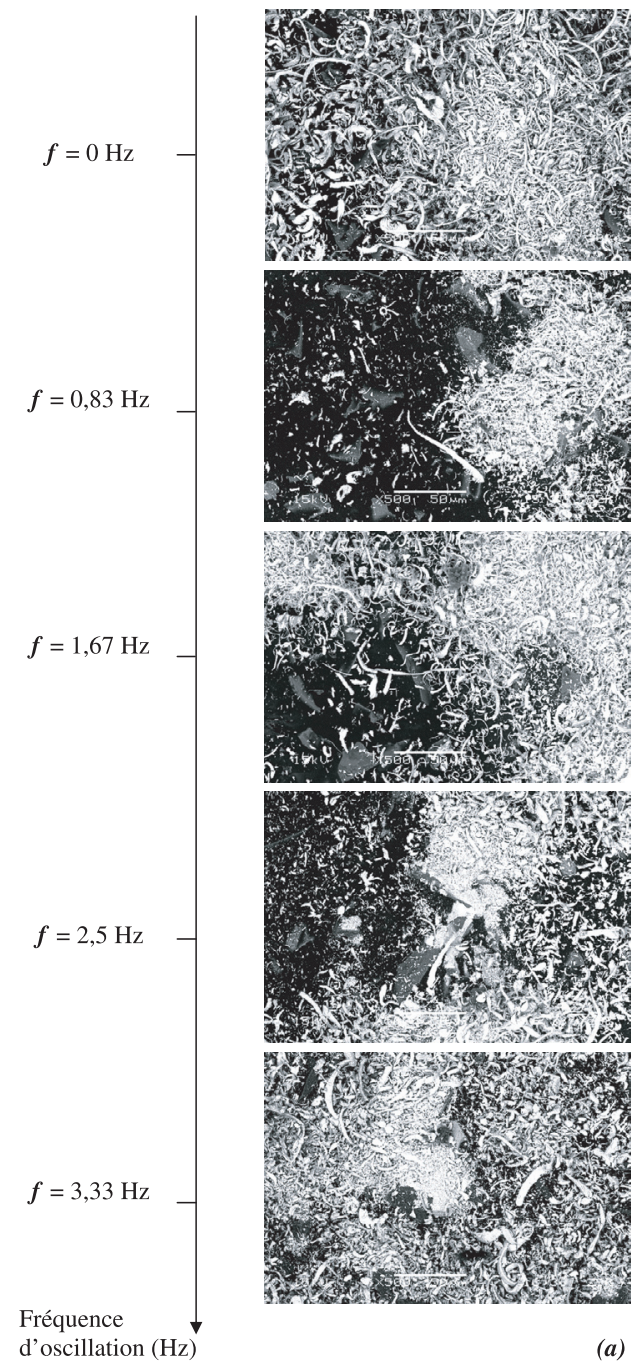

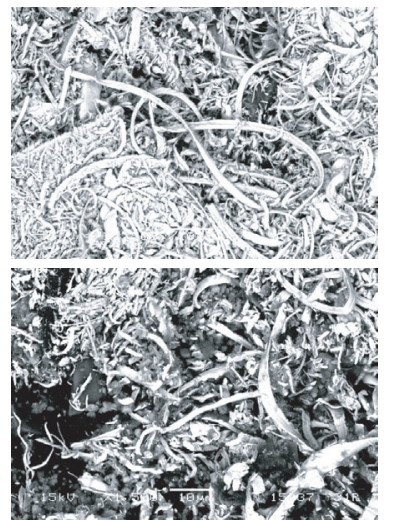
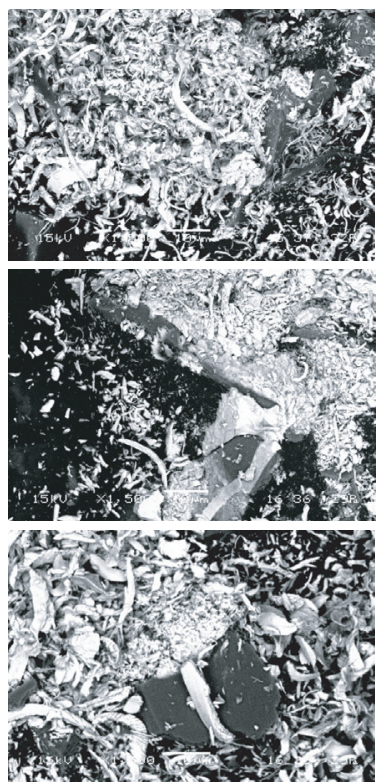

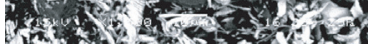

(b)

Fig. 14. Évolution de la couche de micro-débris transférée sur la toile abrasive en fonction de la fréquence des oscillations axiales $f$ (grossissement : $\times 500 \mathrm{a}$ ) et $\times 1500 \mathrm{~b})$; inclinaison des observations : $\left.0^{\circ}\right)$. $\left(t_{\text {toilage }}=9 \mathrm{~s}, N_{\mathrm{r}}=100\right.$ tr.min ${ }^{-1}, F_{\mathrm{s}}=400 \mathrm{~N}$, $A=1 \mathrm{~mm}$, taille des grains abrasifs : $30 \mu \mathrm{m}$, matériau des grains abrasifs : AlO, type mâchoires : rigides.) 

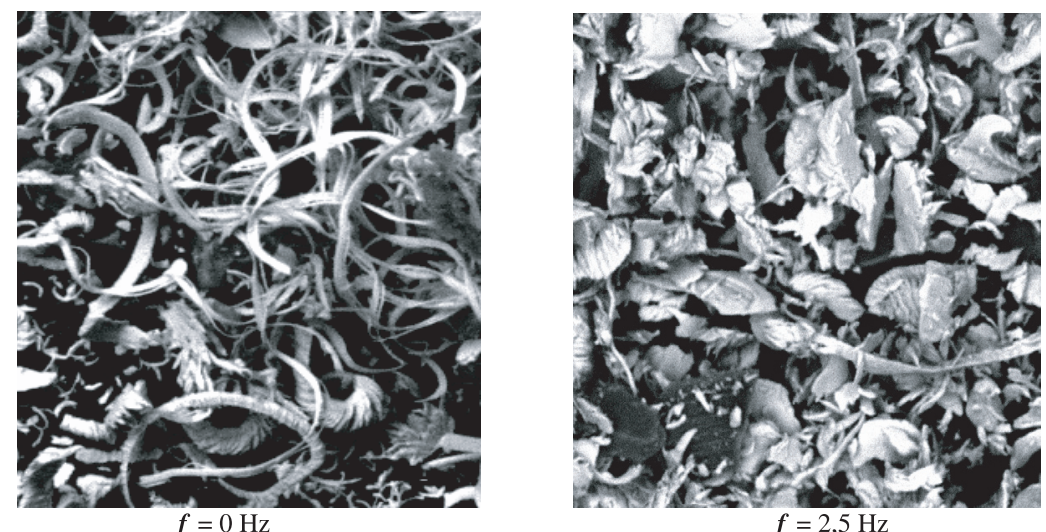

Fig. 15. Morphologie évolutive des micro-copeaux générés par toilage en fonction de la fréquence des oscillations $f$ (grossissement : $\times 1500$; inclinaison des observations : $\left.0^{\circ}\right) .\left(t_{\text {toilage }}=9 \mathrm{~s}, N_{\mathrm{r}}=100 \operatorname{tr} \min ^{-1}, F_{\mathrm{s}}=400 \mathrm{~N}, A=1 \mathrm{~mm}\right.$, taille des grains abrasifs : $30 \mu \mathrm{m}$, matériau des grains abrasifs : AlO, type mâchoires : rigides.)

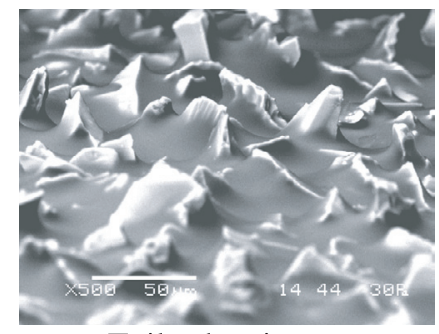

Toile abrasive neuve

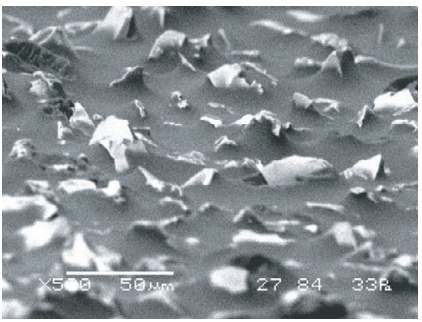

$f=1,66 \mathrm{~Hz}$

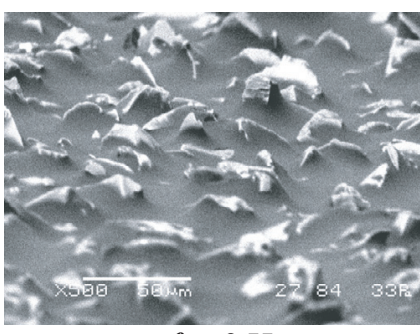

$f=0 \mathrm{~Hz}$

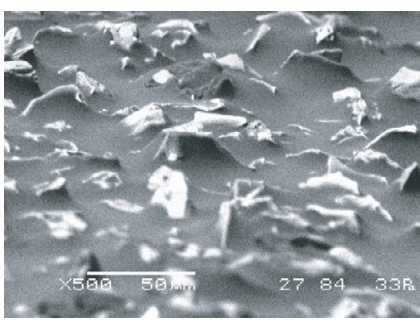

$f=2,5 \mathrm{~Hz}$

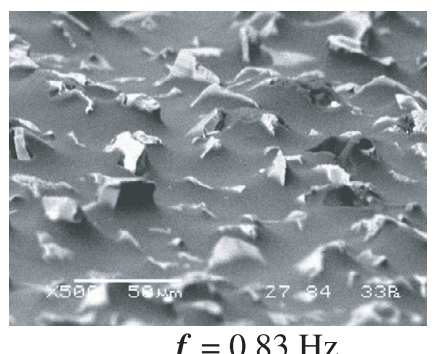

$f=0,83 \mathrm{~Hz}$

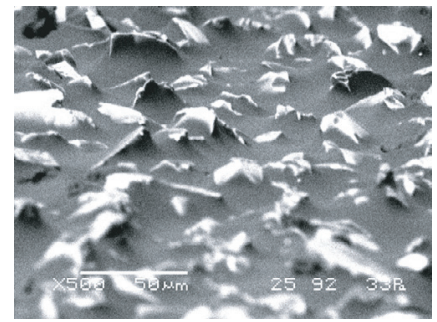

$f=3,33 \mathrm{~Hz}$

Fig. 16. Morphologie évolutive des grains abrasifs de la toile après nettoyage en fonction de la fréquence d'oscillations $f$ $\left(t_{\text {toilage }}=9 \mathrm{~s}, N_{\mathrm{r}}=100 \mathrm{tr} \cdot \mathrm{min}^{-1}, F_{\mathrm{s}}=400 \mathrm{~N}, A=1 \mathrm{~mm}\right.$, taille des grains abrasifs : $30 \mu \mathrm{m}$, matériau des grains abrasifs : AlO, type mâchoires : rigides).

micrographies de la figure 14 ont été réalisées au MEB en mode rétrodiffusé (BSE). Dans ce cas, le contraste est dû à la nature chimique et dépend de la masse du matériau. On distingue ainsi aisément la couche de transfert de la toile : ce qui est blanc est lourd (micro-copeaux), inversement le sombre est léger (la toile). Sans fréquence d'oscillation, la toile est entièrement recouverte d'une couche de micro-débris. Elle est constituée principalement de microcopeaux primaires longs, libres ou logés et issus de la coupe. L'application de l'oscillation axiale de la toile, pour une fréquence donnée, entraîne un recouvrement partiel des micro-débris composés de (cf. Figs. 14 et 15) :

- micro-copeaux primaires fragmentés ;

- micro-copeaux secondaires, courts hélicoïdaux issus de bourrelets latéraux;

- particules broyées et agglomérées.
L'oscillation axiale de la toile implique en effet que les différents pouvoirs de coupe en bout d'un grain puissent opérer en coupe. Dans ce cas, l'influence de la fréquence d'oscillation se manifeste par la prédominance de la coupe qui favorise un processus accéléré de fragmentation des micro-copeaux. Ceci résulte aussi d'un piégeage des débris amoindri sous l'effet vibratoire des oscillations. Là aussi, on note encore une fois de plus le rôle important de l'arrosage pour enlever les déchets de l'outil (toile) et entretenir ainsi le pouvoir de coupe.

Les observations microscopiques de la toile après nettoyage sont résumées par la figure 16. De son analyse, la morphologie évolutive des grains d'abrasif (nouvelle angularité ou émoussage des arêtes vives) ne semble pas être affectée par la fréquence d'oscillation. Ce résultat confirme aussi le rôle protecteur du paramètre d'oscillation pendant le contact répété et alterné entre la toile et la pièce. 


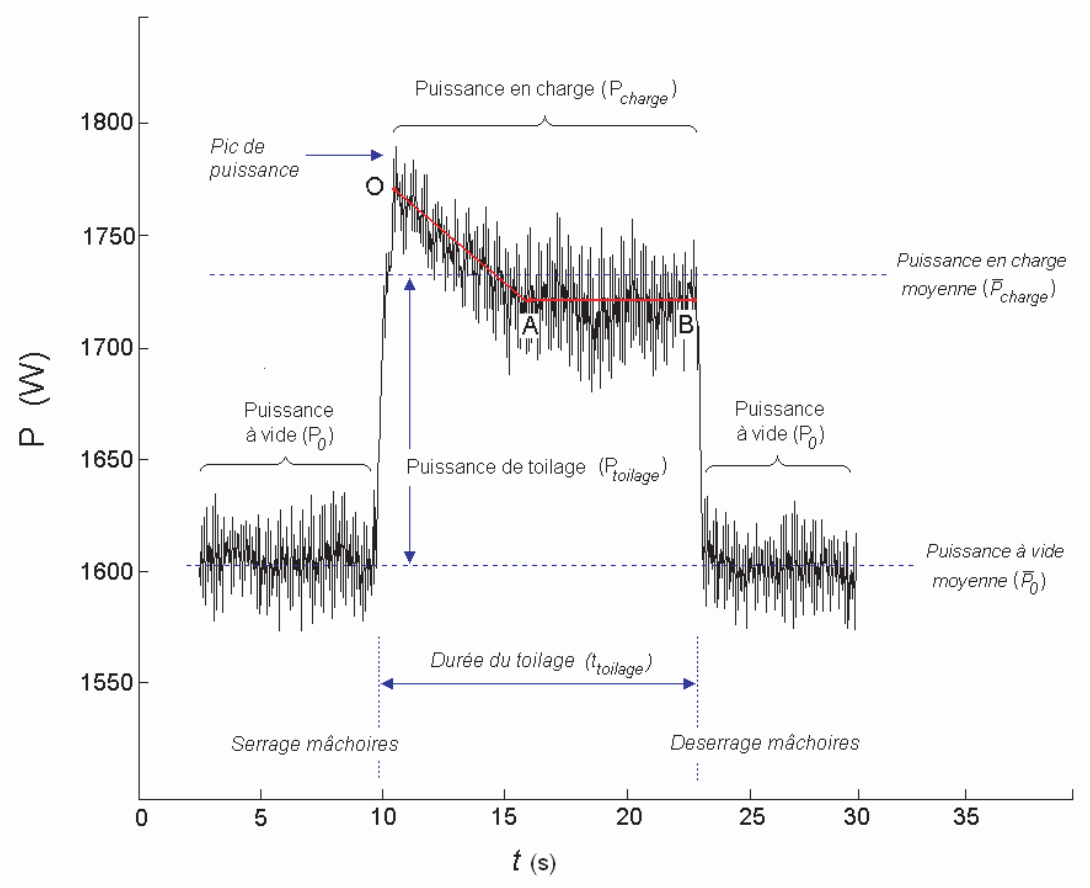

Fig. 17. Courbe typique de l'évolution de la puissance consommée au cours d'une opération de toilage.

\section{Description énergétique de l'opération de toilage}

\subsection{Analyse du test énergétique}

Les courbes de variation de la puissance en charge et à vide en fonction du temps définissent la valeur de la puissance absorbée par le processus de toilage $P_{\text {toilage }}$ (cf. Fig. 17). Celle-ci s'ajoute à la puissance à vide $P_{0}$ dès le serrage des mâchoires, puis progresse en quelques secondes jusqu'à un maximum. Elle décroît ensuite (pente OA sur la Fig. 17) pour se stabiliser (segment AB sur la Fig. 17) pendant le reste de la durée de toi-

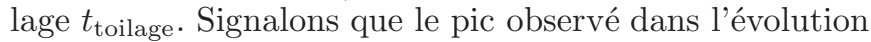
de la puissance en charge se trouve parfois occulté par le temps nécessaire à la fermeture complète des mâchoires.

L'évolution de la puissance absorbée par le toilage $P_{\text {toilage }}$ en fonction du temps de cycle et de la fréquence d'oscillation est reportée sur la figure 18. Il est constaté que celle-ci diminue avec le temps de cycle (cf. Fig. 18a). Cependant aucune variation significative de la puissance consommée n'est observée lorsque la fréquence varie de 0 à $3,3 \mathrm{~Hz}$ (cf. Fig. 18b). En effet, les dispersions importantes sur toute la plage de fréquence étudiée perturbent l'analyse des résultats. À noter que pour $f=2,5 \mathrm{~Hz}$, un minimum de puissance est consommé avec une faible dispersion.

\subsection{Discussion}

Bien évidement, les mécanismes d'enlèvement de matière dans les opérations d'usinage par abrasion sont

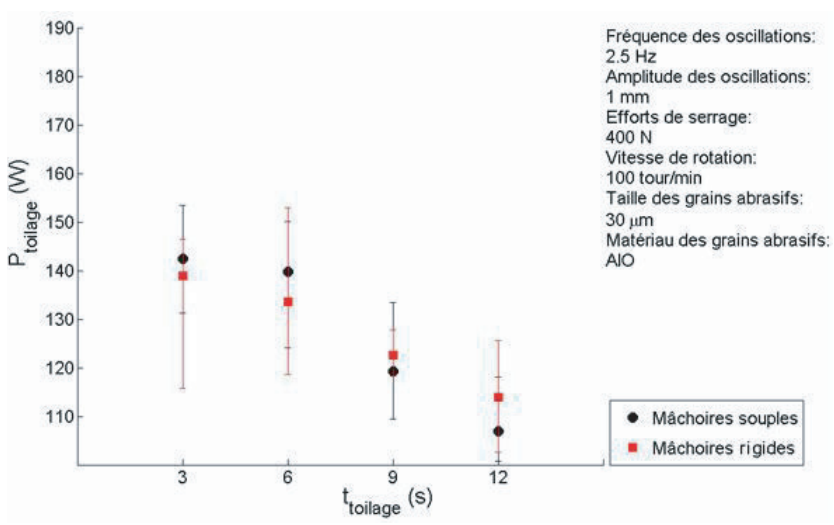

(a)

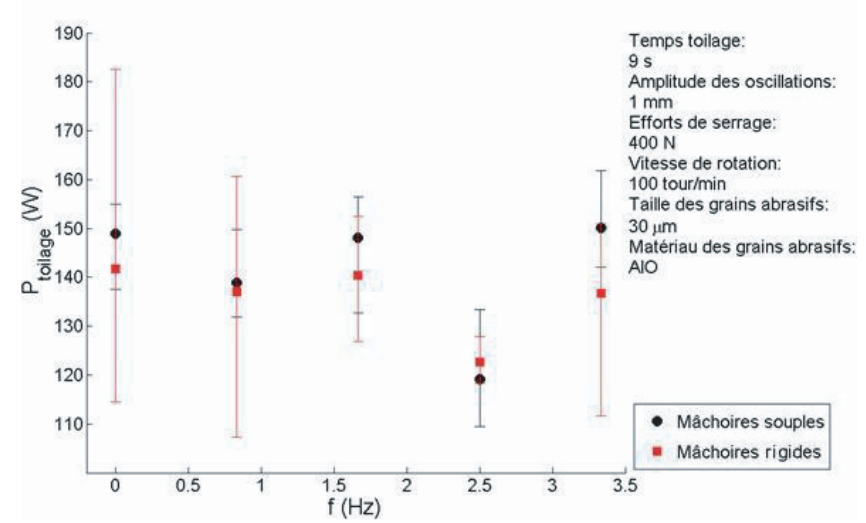

(b)

Fig. 18. Les variations de la puissance de toilage $\left(P_{\text {toilage }}\right)$ en fonction du temps de cycle a) et de la fréquence d'oscillation b) pour les deux mâchoires testées. 


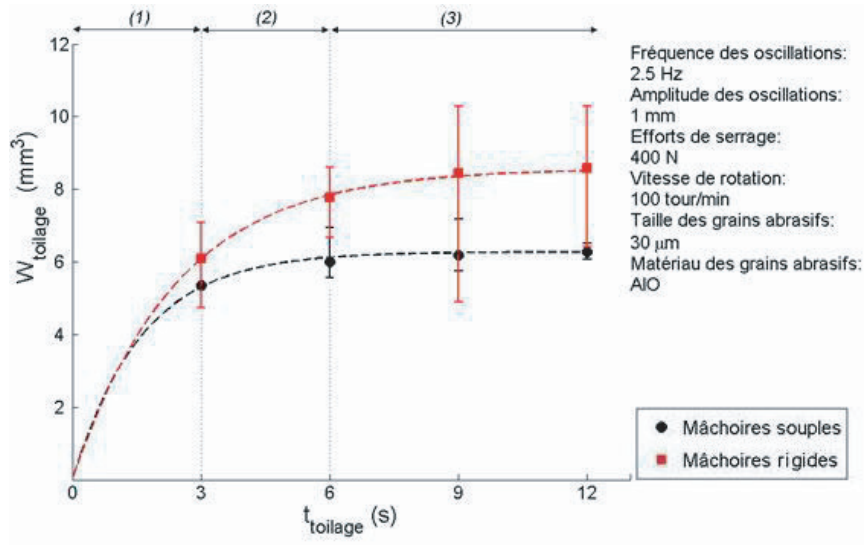

Fig. 19. Évolution de l'usure volumique ( $\left.W_{\text {toilage }}\right)$ des portées toilées avec le temps de cycle ( $\left.t_{\text {toilage }}\right)$.

plus difficiles à appréhender par le praticien, puisque ils s'effectuent à l'échelle microscopique des grains. Cependant, les mesures énergétiques peuvent permettre une qualification du processus de finition en toilage mais, plus encore, ils doivent permettre un traitement de l'information technologique afin d'optimaliser cette opération. Ainsi, si l'on considère l'usure volumique du matériau abrasé, c'est-à-dire le volume de matière enlevée par le processus de toilage, celle-ci évolue avec le temps de cycle comme le montre la figure 19. L'interprétation physique, en terme de mécanismes d'enlèvement de matière, des courbes est possible car on y distingue fort bien :

- la zone (1) avec un micro-enlèvement de matière très important;

- la zone de transition (2) avec la diminution de la composante coupe en toilage;

- la zone de frottement (3) où l'usure volumique n'évolue pas.

Le volume de matière enlevée en toilage dépend également de la dureté des mâchoires de toile : un support de toile souple génère plutôt une régularité microgéométrique qu'un enlèvement de matière (cf. Fig. 19). Pour mieux préciser l'ordre de ces phénomènes qui se superposent en cours de l'opération de toilage, une analyse physique basée sur l'énergie consommée par toilage a été effectuée. La figure 20 montre la variation de celle-ci en fonction de la durée effective de toilage (temps de cycle). Dans cette représentation, on distingue deux régimes de toilage :

- Pour $t_{\text {toilage }}<t_{\mathrm{c}}$ un régime de toilage primaire où le processus de toilage est dominé par la formation de micro-copeaux, mais au prix d'énergies consommées élevées (l'énergie de toilage $E_{\text {toilage }} \approx 400 \mathrm{~J}$ );

- Pour $t_{\text {toilage }}>t_{\mathrm{c}}$ un régime de toilage secondaire (régime établi) où l'opération de toilage s'apparente alors plus à une compétition entre frottement et labourage qu'à un enlèvement de matière $\left(E_{\text {toilage }}\right.$ chute à $250 \mathrm{~J}$ environ).

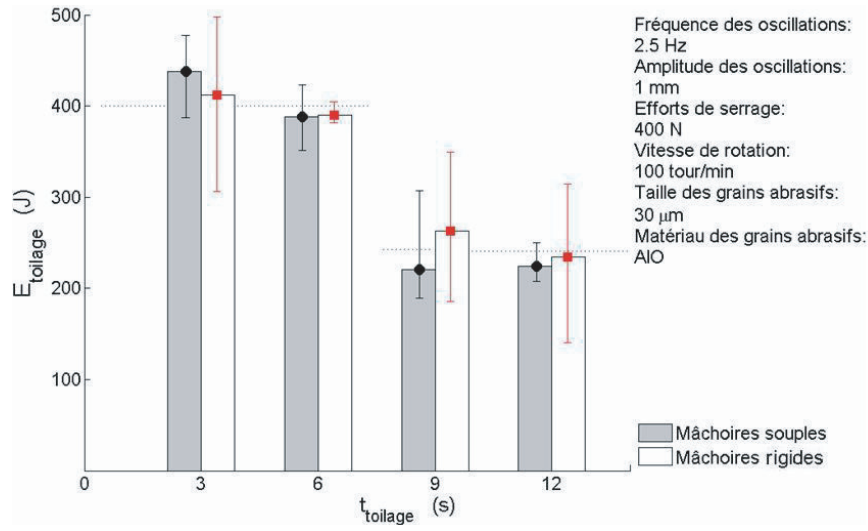

Fig. 20. Évolution de l'énergie consommée par toilage ( $\left.E_{\text {toilage }}\right)$ en fonction du temps de cycle $t_{\text {toilage }}$.

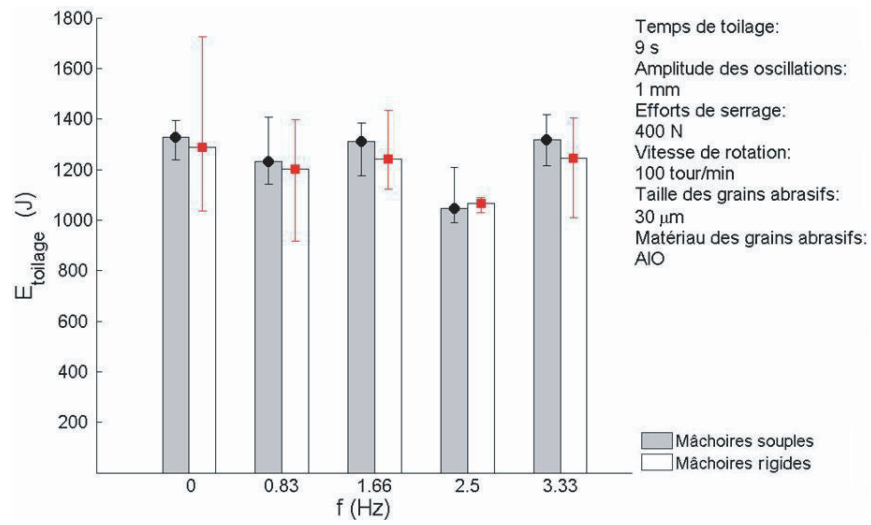

Fig. 21. Énergie consommée par toilage $\left(E_{\text {toilage }}\right)$ en fonction de la fréquence d'oscillation $f$.

À ce stade, on peut déjà noter le rôle joué par le critère intrinsèque de l'énergie consommée dans la qualification des régimes de toilage. Toutefois, à temps de cycle fixe et à fréquence d'oscillation variable, aucun changement de régime de toilage n'est observé (cf. Fig. 21). Le rendement énergétique est stable avec un minimum d'énergie consommée pour $f=2,5 \mathrm{~Hz}$.

D'un point de vue énergétique, le processus de toilage consomme une énergie $E_{\text {toilage }}$ pour produire un débit volumique de matière $Q_{\mathrm{m}}$. L'énergie consommée rapportée au débit de matière produit est, par définition, l'énergie spécifique de toilage $E_{\mathrm{sp}}^{\mathrm{t}}$ qui caractérise son rendement énergétique. Si l'on considère cette variable physique, grandeur homogène à une contrainte, on prend en compte simultanément les effets de coupe, de déformation plastique « labourage » et les effets de frottement.

Les figures 22 et 23 illustrent respectivement l'évolution de l'énergie spécifique de toilage selon le temps de cycle et la fréquence d'oscillation. Il y a lieu de remarquer que l'énergie spécifique de toilage augmente linéairement avec le temps de cycle. Pour le minimum d'énergie à puissance consommée identique, la productivité sera maximale. Sous cet aspect l'énergie spécifique de toilage présente bien un critère global de qualification 


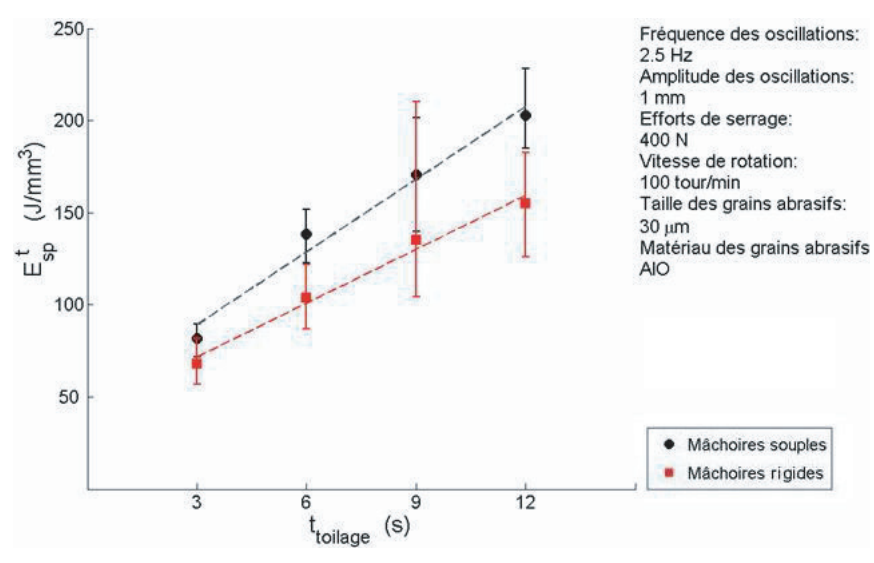

Fig. 22. Évolution de l'énergie spécifique de toilage $\left(E_{\mathrm{sp}}^{\mathrm{t}}\right)$ en fonction du temps de cycle $t_{\text {toilage }}$.

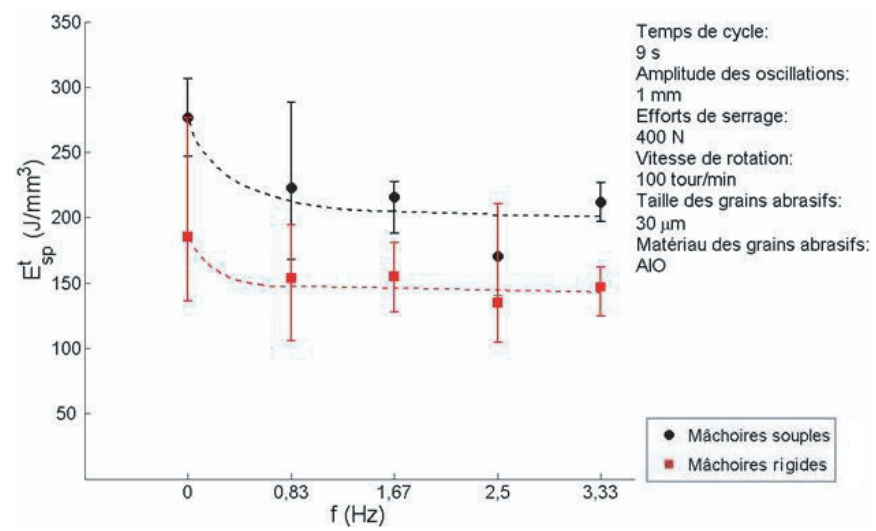

Fig. 23. Évolution de l'énergie spécifique de toilage $\left(E_{\mathrm{sp}}^{\mathrm{t}}\right)$ en fonction de la fréquence d'oscillation $f$.

pour une opération de toilage. Le battement axial de la toile conduit à une réduction de l'énergie spécifique de toilage (cf. Fig. 23). Ceci signifie que l'oscillation de la bande augmente son pouvoir de coupe. Notons néanmoins, que cet effet est indépendant de la fréquence d'oscillation et ce pour la plage de fréquence étudiée.

\section{Conclusions}

De cette étude de l'intervention des variables process de toilage (temps de cycle et fréquence d'oscillation du contact film abrasif/pièce) sur les spécificités d'état de surface et de forme, il ressort que la mise au point des tests de qualification du processus de toilage peut être effectuée à partir de mesures énergétiques. Les résultats du test énergétique couplés aux essais d'expertise du retour d'essais précisent que :

1. L'influence du temps de cycle fait apparaître deux régimes :

- d'une part, un régime primaire de toilage, qualifié de transitoire et caractérisé par un temps limite de coupe $t_{\mathrm{c}}$, où la superfinition de courte durée implique plutôt une dominance par enlèvement de matière (coupe);

- d'autre part, le prolongement du toilage jusqu'à un régime établi, régime secondaire de toilage, induit à la prédominance des composants abrasion à trois corps et frottement.

2. Par oscillation axiale de la bande, il y a diminution du frottement à l'interface grains d'abrasif/micro-copeau et un accroissement du pouvoir de coupe favorisé par une évacuation entretenue des micro-copeaux.

3. L'enroulement total du palier avec la toile, provoqué par la forme des mâchoires de travail, conduit à un piégeage des micro-copeaux par logement et formation, au sens des matériaux, d'un troisième corps qui perturbe la fonctionnalité de la toile abrasive. Ceci rend difficile l'interprétation des effets de la dureté des mâchoires de toile et suggère le rôle primordial à la fin, du liquide de coupe et l'avance de la toile dans l'élimination de ce troisième corps et son évacuation.

Par ailleurs, quels sont les avantages que l'on peut tirer de la méthode énergétique d'analyse des effets des variables « process $» ?$

En toilage, le test énergétique couplé aux expertises de retour d'essai :

- permet de pister l'intervention de chaque variable «process $»$;

- utilise des paramètres accessibles à l'utilisateur ;

- s'applique directement sur les machines de production afin de réaliser des états de référence machine (appelés souvent « $\mathrm{km} 0 »)$ pour l'analyse lors des dérives en production;

- apporte enfin des précisions sur l'influence simultanée des paramètres technologiques et des propriétés physiques des matériaux toile-pièce en présence. C'est cette voie qu'il faut approfondir pour mettre au point un modèle représentatif du procédé de toilage.

Remerciements. Les auteurs remercient les Directions Mécanique et Ingénierie Process de RENAULT pour le financement de cette étude dans le cadre de la convention CIFRE $n^{\circ}: 769 / 2004$.

Les auteurs remercient également les partenaires de l'étude : Service Métiers du Process de RENAULT.

\section{Références}

[1] D. Baudier, La science des frottements, R\&D la route de l'innovation, Le magazine de la recherche et du développement, Renault 33 (2004) 33-37

[2] M.C. Shaw, Principles of Abrasive Processing, Oxford Science Publications, Clarendon Press, Oxford, 1996

[3] H. Kim, K.M. Marsheck, Belt Forces with Grinding, J. Engineering for Industry 110 (1988) 201-211

[4] G. Zambelli, L. Vincent, Matériaux et contact - Une approche tribologique, Presses polytechniques et universitaires romandes, 1998, pp. 89-97

[5] G.W. Stochowik, A.W. Botchelar, Engineering Tribology, Butterworth-Heinemann, 2001 (second edition), pp. $483-507$ 
[6] A. Jourani, M. Dursapt, H. Hamdi, J. Rech, H. Zahouani, Effect of the belt grinding on the surface texture : Modeling of the contact and abrasive wear, Wear 259 (2005) 1137-1143

[7] W. Qu, K. Wang, M.H. Miller, Y. Huang, A. Chandra, Using vibration-assisted grinding to reduce subsurface damage, Precision Engineering 24 (2000) 329-337

[8] D.M. Schibish, U. Friedrich, Technologie de superfinition : Des surfaces de haute précision, Verlag moderne industrie, Supfina Grieshaber, 2002

[9] J. Bielle, Usinage par grains d'abrasif non-libres : choisir la meule ou le film abrasif?, communication privée, 2001
[10] H. Kim, K.M. Marsheck, M. Naji, Force between an abrasive belt and pulley, Mech. Mach. Theory 22 (1987) 97-103

[11] H. Kim, K.M. Marsheck, Force Distribution for a Flat Belt Drive with a Concentrated Contact Load, Mech. Mach. Theory 25 (1990) 667-677

[12] R.W. Story, Forces and Force Ratios in Grinding with Coated Abrasives, J. Engineering for Industry (1968) 407-410

[13] T.K. Puthanangady, S. Malkin, Experimental investigation of the superfinishing process, Wear 185 (1995) 173-182 